\title{
EUROSPINE 2000
}

\section{Abstracts of the Annual Meeting of the Spine Society of Europe}

\section{Contents}

\section{Papers read}

Back pain/osteoporosis

Deformities/scoliosis

Electrophysiology and monitoring

Scoliosis

Basic science I

Cervical spine

Outcome

Basic science II

Surgery

Imaging

New technologies
Paper no.

$1-15$

$16-22$

$23-27$

28-35

$36-42$

$43-49$

$50-56$

$57-63$

64-77

$78-87$

88-94 page

283

288

290

291

294

296

299

301

304

308

311

\section{Posters}

In alphabetical order 